\title{
A phantom node formulation with mixed mode cohesive law for splitting in laminates
}

\author{
F. P. van der Meer • L. J. Sluys
}

Received: 27 October 2008 / Accepted: 8 April 2009 / Published online: 8 May 2009

(C) The Author(s) 2009. This article is published with open access at Springerlink.com

\begin{abstract}
A phantom node method with mixed mode cohesive law is proposed for the simulation of splitting in laminates. With this method, a discontinuity in the displacement field can be modeled at arbitrary locations. The micromechanical phenomenon that splitting cracks grow parallel to the fiber, is incorporated on the mesolevel, i.e., in the homogenized ply, by setting the direction of the crack propagation equal to the fiber direction. A new mixed mode cohesive law is introduced for increased robustness of the incremental-iterative solution procedure. The model is validated with mixed mode bending tests, and its utility is illustrated with examples for a single ply and for a laminate.
\end{abstract}

Keywords Composite laminates - Progressive failure $\cdot$ Splitting $\cdot$ Phantom node method $\cdot$ Mixed mode $\cdot$ Cohesive zone model

\section{Introduction}

Failure in composite laminates may involve different processes such as fiber fracture, splitting, distributed matrix cracking, delamination and fiber kinking. Moreover, it has been observed in experiments that the type of failure that is observed in simple tests depends on the specimen size (Hallett and Wisnom 2006a; Green et al.

F. P. van der Meer $(\varangle) \cdot$ L. J. Sluys

Faculty of Civil Engineering and Geosciences, Delft

University of Technology, P.O. Box 5048, 2600 GA Delft,

The Netherlands

e-mail: f.p.vandermeer@tudelft.nl
2007; Wisnom et al. 2008). This makes prediction of strength and toughness of a laminate with a particular geometry and layup a demanding job. There is a need for numerical models for each of the relevant processes, which, when combined, allow for reliable analysis of progressive failure in laminates.

Numerical analysis of laminates is often endeavored at mesolevel, which means that every ply is modeled as a homogeneous continuum, with orthotropic material properties related to the directions of the fibers in the ply. Delamination, i.e., failure between the plies, can then be modeled with interface elements (e.g., Turon et al. 2006; Yang and Cox 2005). For failure inside the ply, numerous formulations have been proposed for stress based failure criteria, ranging from classical criteria by Tsai and Wu (1971) and Hashin (1980) to more recent ones by Puck and Schürmann (1998, 2002) and Dávila et al. (2005). These strength criteria can be used in progressive failure analysis, when they are extended with material degradation laws. For this purpose, several continuum damage models have been developed (e.g., Lapczyk and Hurtado 2007; Laurin et al. 2007; Maimí et al. 2007; Matzenmiller et al. 1995; Pinho et al. 2006). However, it can be doubted whether the continuum models are suitable to capture the particular phenomenon of splitting correctly (van der Meer and Sluys 2009). The reason for this is illustrated in Fig. 1. In the real material, failure will be concentrated in the weaker matrix domain, while homogenized continuum material models do not offer this domain separation. 


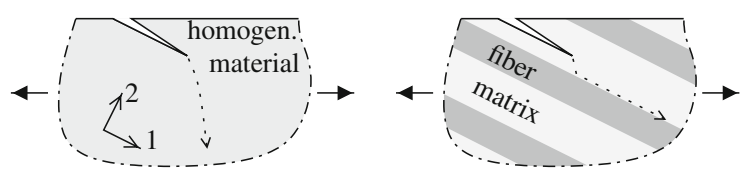

Fig. 1 Crack propagation in an homogeneous orthotropic medium and in a fiber-matrix material

Therefore, a discontinuous approach to modeling failure is to be preferred for the representation of splitting. When a split is modeled as a discontinuity, it is possible to fix the direction of propagation. Thus, the micromechanical phenomenon that ply cracks tend to propagate in fiber direction can be incorporated at mesolevel. One way to implement this is to model the split with interface elements (see Wisnom and Chang 2000; Yang and Cox 2005; Hallett and Wisnom 2006b; Jiang et al. 2007). In that case, however, cracks may only appear between elements, which may complicate mesh generation. Moreover, the location of cracks must be specified in advance, which limits the predictive capability of the model, except when interface elements are placed throughout the specimen, which would aggravate the meshing difficulties and lead to a significant increase in the number of nodes.

With the phantom node method, it is possible to model discontinuities at an arbitrary location in the mesh. Following the work by Hansbo and Hansbo (2004), Mergheim et al. (2005) and Song et al. (2006), a crack is introduced by addition of an extra element on top of an existing element. It has been shown (Song et al. 2006), that this method is equivalent to the extended finite element method (XFEM) in which a discontinuity in the displacement field is introduced by enrichment of the shape functions with the Heaviside step function (e.g., Wells and Sluys 2001; Moës and Belytschko 2002). While XFEM is suitable for additional enrichment of the displacement field around the crack tip to capture the singular field (Belytschko and Black 1999; Moës et al. 1999), the phantom node method is only applicable to cohesive crack modeling where the singularity in the stress field is removed due to the presence of a cohesive traction. It has been shown by Rabczuk et al. (2008) how the phantom node can be extended to model the crack tip inside an element, but here the simpler approach is followed, in which the discontinuity grows elementwise and the tip is always located at an element boundary.

Another variant to XFEM has been applied in the context of splitting in composite laminates by Iarve
(2003) and Mollenhauer et al. (2006). Higher order shape functions were used, instead of the Heaviside step function, to approximate the discontinuity in the displacement field. This method was applied for detailed analysis of the stress field in laminates in the presence of splitting cracks. However, crack propagation and initiation was not discussed.

In the analysis of mixed mode cohesive cracks, the highly nonlinear nature of the traction-separation law endanger the robustness of the computation. Moonen et al. (2008) have introduced a new formulation for cohesive tractions in which this nonlinearity is reduced significantly. In this paper, this cohesive traction law is adapted such that the energy dissipated in mixed mode fracture can be given as a function of the mode ratio. For this function, the interaction law proposed by Benzeggagh and Kenane (1996) is used.

This paper is organized as follows. First the phantom node method is presented. Then the formulation of the cohesive law is treated, followed by a description of the loading strategy with a dissipation-based arclength method. The cohesive law is validated with the simulation of mixed mode bending tests. Furthermore, the superiority of the approach over continuum models is illustrated with the example of an off-axis tensile test. Finally, results are presented from an analysis of a cross ply laminate in which splitting interacts with delamination. With this last example, the usability of the method in laminate analysis is demonstrated.

\section{Phantom node method}

In this section, the phantom node method for discontinuous representation of cracks including crack initiation and propagation is presented.

\subsection{Kinematical relations}

Consider an element in a finite element mesh with nodes $n_{1}, \ldots, n_{4}$ (see Fig. 2). This element is crossed by a crack at $\Gamma_{c}$, dividing the element domain into two complementary subdomains, $\Omega_{A}$ and $\Omega_{B}$. In the phantom node method, a discontinuity in the element displacement field is constructed by adding phantom nodes (here labeled $\tilde{n}_{1}, \ldots, \tilde{n}_{4}$ ) on top of the existing nodes. The existing element is replaced by two new elements, referred to as element $A$ and element $B$. The connectivity of these overlapping elements is 
Fig. 2 Connectivity and active parts of two overlapping elements in phantom node method
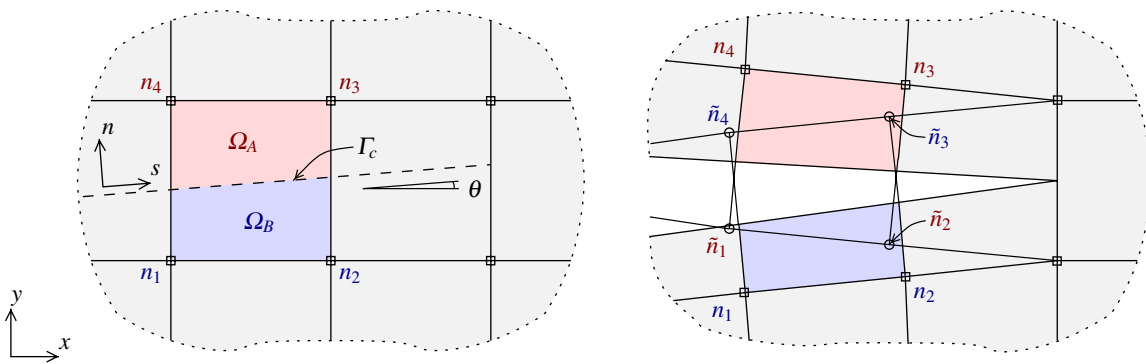

$\operatorname{nodes}_{A}=\left[\tilde{n}_{1}, \tilde{n}_{2}, n_{3}, n_{4}\right]$

$\operatorname{nodes}_{B}=\left[n_{1}, n_{2}, \tilde{n}_{3}, \tilde{n}_{4}\right]$

The elements do not share nodes, and therefore have independent displacement fields. Both elements are only partially active, the active part of element $A$ is $\Omega_{A}$ and the active part of element $B$ is $\Omega_{B}$. This is represented numerically in the definition of the displacement field: the displacement of a point with coordinates $\mathbf{x}$ is computed with the standard finite element shape functions $\mathbf{N}(\mathbf{x})$ and the nodal displacement values from either of the overlapping elements, depending on the location of the point:

$\mathbf{u}(\mathbf{x})= \begin{cases}\mathbf{N}(\mathbf{x}) \mathbf{u}_{A}, & \mathbf{x} \in \Omega_{A} \\ \mathbf{N}(\mathbf{x}) \mathbf{u}_{B}, & \mathbf{x} \in \Omega_{B}\end{cases}$

The strain field is derived from the displacement field in common fashion:

$\boldsymbol{\varepsilon}(\mathbf{x})= \begin{cases}\boldsymbol{\varepsilon}_{A}(\mathbf{x})=\mathbf{B}(\mathbf{x}) \mathbf{u}_{A}, & \mathbf{x} \in \Omega_{A} \\ \boldsymbol{\varepsilon}_{B}(\mathbf{x})=\mathbf{B}(\mathbf{x}) \mathbf{u}_{B}, & \mathbf{x} \in \Omega_{B}\end{cases}$

where $\mathbf{B}(\mathbf{x})$ is the strain nodal displacement matrix $\mathbf{B}(\mathbf{x})=\mathbf{L N}(\mathbf{x})$ with

$\mathbf{L}^{T}=\left[\begin{array}{ccc}\frac{\partial}{\partial x} & 0 & \frac{\partial}{\partial y} \\ 0 & \frac{\partial}{\partial y} & \frac{\partial}{\partial x}\end{array}\right]$

The displacement jump over the crack is defined as the difference between the displacement fields of the two elements.

$\llbracket \mathbf{u} \rrbracket(\mathbf{x})=\mathbf{N}(\mathbf{x})\left(\mathbf{u}_{A}-\mathbf{u}_{B}\right), \quad \mathbf{x} \in \Gamma_{c}$

The displacement jump can be expressed in the local coordinate frame with normal and shear components as

$\llbracket \overline{\mathbf{u}} \rrbracket=\left\{\llbracket u \rrbracket_{n} ; \llbracket u \rrbracket_{s}\right\}^{T}=\mathbf{Q} \llbracket \mathbf{u} \rrbracket$

where

$\mathbf{Q}=\left[\begin{array}{cc}-\sin \theta & \cos \theta \\ \cos \theta & \sin \theta\end{array}\right]$
Closure of the crack tip is enforced when no phantom nodes are added on the element boundary that contains the tip (see Fig. 2). Here the overlapping elements do share nodes, and therefore their displacement fields are not completely independent. When the crack propagates through the next element, phantom nodes are added on top of those nodes that define the element boundary on which the tip was previously located and the connectivity of all cracked elements that contain these nodes is adapted accordingly.

Because the element connectivity is defined such that the old nodes are in the active part of the element and the phantom nodes are in the non-active part, the connectivity of neighboring elements that are not cracked does not have to be adapted.

\subsection{Discretized weak from}

The weak form of the momentum equation for quasistatic analysis in the absence of body forces is written in Voigt notation as:

$\int_{\Omega_{b}} \delta \boldsymbol{\varepsilon}^{T} \boldsymbol{\sigma} \mathrm{d} \Omega+\int_{\Gamma_{c}} \delta \llbracket \mathbf{u} \rrbracket^{T} \mathbf{t} \mathrm{d} \Gamma=\int_{\Gamma_{N}} \delta \mathbf{u}^{T} \mathbf{t}_{N} \mathrm{~d} \Gamma$

where $\Omega_{b}$ is the bulk domain, $\Gamma_{c}$ is the cracked surface and $\Gamma_{N}$ is the surface along which Neumann boundary conditions are applied. The cohesive traction is denoted $\mathbf{t}$ and the external traction is denoted $\mathbf{t}_{N}$.

The weak form is discretized with standard Galerkin procedures. The assembly of the internal force vector is executed elementwise, where the contribution from uncracked elements is standard:

$\mathbf{f}_{(\mathrm{el})}^{\mathrm{int}}=\int_{\Omega_{(\mathrm{el})}} \mathbf{B}^{T} \boldsymbol{\sigma}(\boldsymbol{\varepsilon}) \mathrm{d} \Omega$

in which $\sigma(\varepsilon)$ is a continuum constitutive law. For the examples in this paper, an orthotropic linear elastic constitutive law for plane stress is used:

$\sigma(\varepsilon)=\mathbf{D} \varepsilon$ 
with

$$
\mathbf{D}=\mathbf{R}^{T} \overline{\mathbf{D}} \mathbf{R}
$$

and

$$
\mathbf{R}=\left[\begin{array}{ccc}
\cos ^{2} \theta & \sin ^{2} \theta & \sin \theta \cos \theta \\
\sin ^{2} \theta & \cos ^{2} \theta & -\sin \theta \cos \theta \\
-2 \sin \theta \cos \theta & 2 \sin \theta \cos \theta & \cos ^{2} \theta-\sin ^{2} \theta
\end{array}\right]
$$

$$
\overline{\mathbf{D}}=\left[\begin{array}{ccc}
\frac{1}{E_{1}} & -\frac{v_{12}}{E_{1}} & 0 \\
-\frac{v_{12}}{E_{1}} & \frac{1}{E_{2}} & 0 \\
0 & 0 & \frac{1}{G_{12}}
\end{array}\right]^{-1}
$$

where $E_{1}$ is the ply stiffness in fiber direction, $E_{2}$ is the transverse ply stiffness, $v_{12}$ is the in plane Poisson's ratio, $G_{12}$ is the in plane shear modulus and $\theta$ is the angle between the $x$-axis and the fiber direction, which is in the current context the same angle as that in Fig. 2 and Eq. 7.

For the cracked elements, the contribution to the internal force vector is less standard. For both overlapping elements, there is a contribution corresponding with both terms on the left hand side of Eq. 8 .

$\mathbf{f}^{\text {int }}=\mathbf{f}^{\text {bulk }}+\mathbf{f}^{\text {coh }}$

For the first contribution, i.e., the contribution from the bulk stress, the definition of strain in Eq. 3 implies that both elements should only be integrated over their active part. Apart from the integration domain, the contribution is similar to that for uncracked elements in Eq. 9:

$$
\begin{aligned}
& \mathbf{f}_{A}^{\text {bulk }}=\int_{\Omega_{A}} \mathbf{B}^{T} \boldsymbol{\sigma}\left(\boldsymbol{\varepsilon}_{A}\right) \mathrm{d} \Omega \\
& \mathbf{f}_{B}^{\text {bulk }}=\int_{\Omega_{B}} \mathbf{B}^{T} \boldsymbol{\sigma}\left(\boldsymbol{\varepsilon}_{B}\right) \mathrm{d} \Omega
\end{aligned}
$$

The second contribution, i.e., the contribution from the cohesive traction, is defined as

$$
\begin{aligned}
\mathbf{f}_{A}^{\mathrm{coh}} & =\int_{\Gamma_{c}} \mathbf{N}^{T} \mathbf{t}(\llbracket \mathbf{u} \rrbracket) \mathrm{d} \Gamma \\
\mathbf{f}_{B}^{\mathrm{coh}} & =-\int_{\Gamma_{c}} \mathbf{N}^{T} \mathbf{t}(\llbracket \mathbf{u} \rrbracket) \mathrm{d} \Gamma
\end{aligned}
$$

where the traction $\mathbf{t}$ is a function of the displacement jump $\llbracket \mathbf{u} \rrbracket$. The exact form of this law will be presented Sect. 3, along with the linearization of the internal force vector. The difference in sign between the two expressions in (16) is related to the definition of $\llbracket \mathbf{u} \rrbracket$ in Eq. 5 and the presence of $\delta \llbracket \mathbf{u} \rrbracket$ in Eq. 8 .

\subsection{Crack initiation and propagation}

For the modeling initiation or propagation of cracks with XFEM or the phantom node method, generally two criteria are needed.

- A criterion to decide whether an element is cracking

- A criterion to assess in which direction the crack grows.

In the particular context of splitting in laminates, the second criterion becomes trivial, because the propagation direction is fixed, viz. equal to the fiber direction. To test for propagation, the stress in the tip element is checked with a stress criterion.

$f(\sigma)=\sqrt{\left(\frac{\left\langle\sigma_{2}\right\rangle}{F_{2 \mathrm{t}}}\right)^{2}+\left(\frac{\tau_{12}}{F_{12}}\right)^{2}}$

in which $\sigma_{2}$ is the transverse stress, $F_{2 \mathrm{t}}$ is the transverse tensile strength, $\tau_{12}$ is the in plane shear stress, and $F_{12}$ is the in plane shear strength. These stress quantities are components of the material stress vector, which is computed as

$\overline{\boldsymbol{\sigma}}=\overline{\mathbf{D}} \mathbf{R} \boldsymbol{\varepsilon}$

The criterion $f(\boldsymbol{\sigma})=1$ is the plane stress equivalent the criterion for tensile matrix failure proposed by Hashin (1980). With the operator $\langle\cdot\rangle=\max \{\cdot, 0\}$ compressive stresses are neglected.

The failure criterion (17) is evaluated in all integration points in critical elements. ${ }^{1}$ The maximum value that is found is checked with the criterion $f(\sigma) \leq 1$. If this criterion is violated, a new crack segment is introduced through this element. In laminate analysis, this check is executed for the maximum value in each ply, therefore cracks may propagate concurrently in different plies.

The following actions are taken when a new crack segment is introduced:

- A new element is added on top of the existing element.

- A numerical integration scheme for both overlapping elements and the cohesive zone is constructed (see Fig. 3). Delaunay triangulation is used, with three integration points per triangle. Because a linear constitutive law is used for the bulk material, no transfer of history variables is needed.

\footnotetext{
$\overline{1}$ In cases with a single crack, there is only one critical element, i.e., the element ahead of the crack tip. When multiple cracking is allowed, all elements that are not too close to existing cracks are critical.
} 

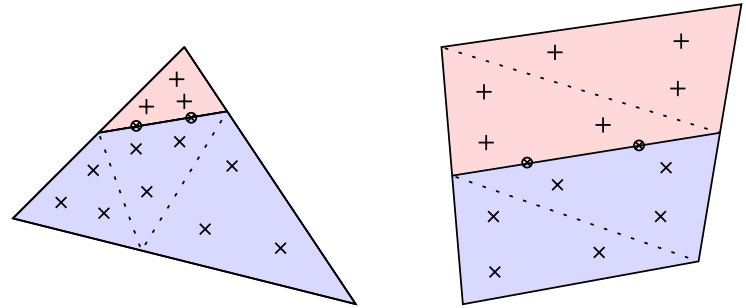

+ Bulk integration point (element $A$ )

$\times$ Bulk integration point (element $B$ )

$\otimes$ Cohesive integration point

Fig. 3 Integration schemes for a pair of overlapping triangular or quadrilateral elements

- Phantom nodes are added on top of the nodes that previously contained the crack tip.

- The element connectivity is updated where needed with respect to the newly added nodes.

The solution algorithm is summarized in Fig. 4. The core of the algorithm is a standard Newton-Raphson iterative procedure to find equilibrium (indicated with solid lines). When the relative norm of the residual vector is sufficiently small, the iterative procedure is considered to have converged. Then the equilibrium solution is checked for failure as described above. When failure occurs, an existing crack is extended or a new crack is introduced, depending on the location of failure. After that, the Newton-Raphson loop is re-entered. The previous converged solution, which satisfied equilibrium but violated the failure criterion, is not dis- carded since this is a better starting point for the iterative procedure than the final converged solution from the previous time step. This is repeated until an equilibrium solution is obtained which does not violate the failure criterion in any location where crack initiation or propagation is allowed. This solution then is the final solution for this time step.

However, when the Newton-Raphson procedure does not converge within a limited number of iterations, the load increment $\Delta \mathbf{f}$ is reduced. Then we go back to the very beginning of the time step, which includes that changes that have been made to the model during this time step (addition of elements and nodes, as well as changes in integration scheme and connectivity) are canceled. Before the next time step is entered, the load increment is adapted, in order to keep time steps as large as possible throughout the computation. The increment is only updated when no crack propagation has occurred. The new increment size is based on the number of iterations (cf. Verhoosel et al. 2008).

The solution algorithm presented in Fig. 4 and explained above is for load control. For the examples presented in this paper, however, an algorithm was used with alternating load and dissipation-based arclength control (Verhoosel et al. 2008). When arclength control is used, the Newton-Raphson loop is slightly different. Furthermore, instead of reduction of $\Delta \mathbf{f}$, reduction of the dissipation increment may be applied, or in some cases switching between the two loading methods. However, the interaction between Newton-Raphson loop, crack propagation loop and adaptive time stepping loop remains essentially the same. In Sect. 4,
Fig. 4 Solution algorithm for single time step with adaptive time stepping and crack propagation under load control

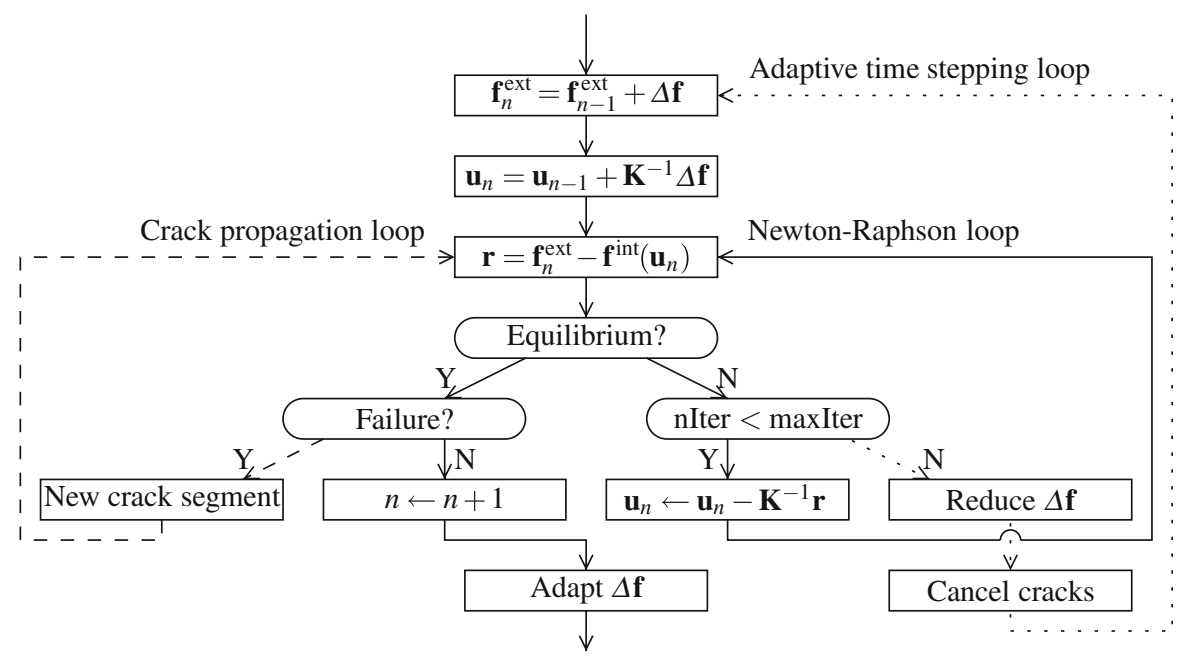


more attention will be given to the implemented loading strategy.

\section{Mixed mode cohesive law}

There is a difficulty in the application of a direct traction separation law for mixed mode cracking. The problem stems from the fact that the traction is not uniquely defined for zero crack opening; in a uniaxial case it is obvious that the traction should be equal to the strength, but in a mixed mode formulation it can be either equal to the normal strength with no shear traction, or to the shear strength with no normal traction, or something in between. The traction evaluation itself remains feasible, because the crack opening will not be exactly equal to zero. However, the highly nonlinear nature of the traction separation law endangers the stability of the Newton-Raphson procedure. Very small variations in nodal displacements give rise to large changes in nodal forces and also, more critically, to large changes in the stiffness matrix.

However, more knowledge on the tractions is available. Equilibrium demands that the ratio between the tensile and shear components of the cohesive traction reflects the ratio between the components of the continuum stress in the bulk material next to the crack. This is taken into account in a cohesive law that was introduced by Moonen et al. (2008), in which the bulk stress is used besides the displacement jump for evaluation of the traction.

In this section, an adapted form of this law is presented. In this new version, the fracture energy as a function of the mode ratio is considered an essential material property.

\subsection{Damage law}

We start from a discrete rigid-damage law (Oliver 2000), written as

$\mathbf{t}=\frac{1-\omega}{\omega} T \llbracket \mathbf{u} \rrbracket$

in which $\omega$ is a damage variable that evolves from 0 to 1 during crack opening and $T$ is a stiffness parameter.In this formulation, the traction is not well-defined upon crack initiation, i.e., when $\omega=0$ and $\llbracket \mathbf{u} \rrbracket=\mathbf{0}$.

Therefore we rewrite it, following Moonen et al. (2008), such that the bulk stress is taken into account.

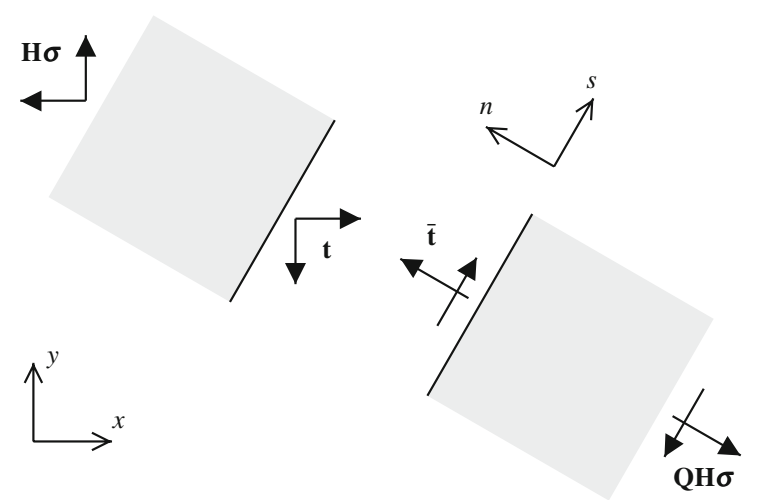

Fig. 5 Cohesive traction and corresponding bulk stress components in local and global coordinate system

Equation 19 is reformulated by multiplication with $\omega$ and addition of $(1-\omega) \mathbf{t}$, which gives:

$\mathbf{t}=(1-\omega)\{\mathbf{t}+T \llbracket \mathbf{u} \rrbracket\}$

Next, we use an equilibrium relation. Namely that the cohesive traction acting on the crack surface must be in equilibrium with the stress in the bulk material next to the crack (see Fig. 5):

$\mathbf{t}=\mathbf{H} \boldsymbol{\sigma}$

where pre-multiplication with $\mathbf{H}$ is the representation in Voigt notation of multiplication of the stress tensor with the normal vector, and

$\mathbf{H}=\left[\begin{array}{ccc}-\sin \theta & 0 & \cos \theta \\ 0 & \cos \theta & -\sin \theta\end{array}\right]$.

Equation 21 is substituted in the right hand side of Eq. 20 which results in the following traction law that is always uniquely defined:

$\mathbf{t}=(1-\omega)\{\mathbf{H} \boldsymbol{\sigma}+T \llbracket \mathbf{u} \rrbracket\}$

We define the effective traction as the undamaged traction vector in the local coordinate system:

$\mathbf{t}^{\mathrm{eff}}=\left\{t_{n}^{\mathrm{eff}} ; t_{s}^{\text {eff }}\right\}^{T}=\mathbf{Q}\{\mathbf{H} \boldsymbol{\sigma}+T \llbracket \mathbf{u} \rrbracket\}$

The rotation with $\mathbf{Q}$ (see Eq. 7) is introduced so that distinction can be made between normal and shear crack opening. Notably, since the crack is parallel to the fiber, the vector $\mathbf{Q H} \boldsymbol{\sigma}$ contains the material stress components $\sigma_{2}$ and $\tau_{12}$. In order to prevent interpenetration of the cracked parts, the damage variable is replaced with a damage tensor. The traction in local coordinate system is defined as:

$\overline{\mathbf{t}}=\left\{t_{n} ; t_{s}\right\}^{T}=[\mathbf{I}-\mathbf{\Omega}] \mathbf{Q}\{\mathbf{H} \boldsymbol{\sigma}+T \llbracket \mathbf{u} \rrbracket\}$ 
with

$\boldsymbol{\Omega}=\omega\left[\begin{array}{cc}\frac{\left\langle t_{n}^{\mathrm{eff}}\right\rangle}{t_{n}^{\mathrm{eff}}} & 0 \\ 0 & 1\end{array}\right]$

Introduction of the factor $\left\langle t_{n}^{\text {eff }}\right\rangle / t_{n}^{\text {eff }}$, which is equal to one when $t_{n}^{\text {eff }}>0$, and equal to zero otherwise, suffices to prevent interpenetration, ${ }^{2}$ because with this factor, $t_{n}^{\text {eff }}<0 \Rightarrow t_{n}=t_{n}^{\text {eff }}$, and, with the assumption of equilibrium $\left(t_{n}=\sigma_{2}\right)$, this gives in turn $t_{n}=$ $t_{n}^{\text {eff }} \Rightarrow \llbracket u \rrbracket_{n}=0$. Because equilibrium is only weakly met, limited interpenetration may occur, but this can be expected to vanish upon mesh refinement.

The final formulation for the cohesive law is obtained after rotating this traction back to the global coordinate system:

$\mathbf{t}=\mathbf{Q}^{T}[\mathbf{I}-\mathbf{\Omega}] \mathbf{Q}\{\mathbf{H} \boldsymbol{\sigma}+T \llbracket \mathbf{u} \rrbracket\}$

During the failure process, the effective traction increases. The damage evolution is driven by a state variable, denoted $\kappa$, which is the maximum history value of a scalar measure for the magnitude of the effective traction:

$\kappa(\tau)=\max _{t \leq \tau} \sqrt{\left(\frac{\left\langle t_{n}^{\mathrm{eff}}\right\rangle}{F_{2 \mathrm{t}}}\right)^{2}+\left(\frac{t_{s}^{\mathrm{eff}}}{F_{12}}\right)^{2}}$

This state variable is used for the evolution of damage variable $\omega$ :

$\omega=\left\{\begin{array}{cc}\frac{\kappa^{f}(\kappa-1)}{\kappa\left(\kappa^{f}-1\right)}, & \kappa<\kappa^{f} \\ 1, & \kappa \geq \kappa^{f}\end{array}\right.$

which results in a linear softening relation between traction and separation. The derivation of the expression for $\kappa^{f}$ will be outlined in Sect. 3.2.

The definition of $\kappa$ in Eq. 28 corresponds with the failure criterion in Eq. 17. As such, continuity in the response of the cracking element is ensured (exactly when the stress in the element is homogeneous, and approximately otherwise). Upon crack initiation, the stress in the element satisfies $f(\sigma)=1$, and the displacement jump $\llbracket \mathbf{u} \rrbracket$ is equal to $\mathbf{0}$. In the closed crack, $\mathbf{t}^{\text {eff }}=\mathbf{Q H} \boldsymbol{\sigma}$, which gives $\kappa=f(\boldsymbol{\sigma})=1$ and $\omega=0$ and consequently $\mathbf{t}=\mathbf{H} \sigma$.

In finite elements, the bulk stress at $\Gamma_{c}$, which is used in the evaluation of the effective traction, is not

\footnotetext{
2 Provided that rotations remain small.
}

uniquely defined. We use the averaged strain from both overlapping elements:

$\sigma=\sigma\left(\varepsilon_{\Gamma_{c}}\right)$

with

$$
\begin{aligned}
\boldsymbol{\varepsilon}_{\Gamma_{c}} & =\frac{1}{2}\left(\boldsymbol{\varepsilon}_{\Gamma_{c}^{+}}+\boldsymbol{\varepsilon}_{\Gamma_{c}^{-}}\right) \\
& =\frac{1}{2} \mathbf{B}\left(\mathbf{x}_{\Gamma_{c}}\right)\left(\mathbf{u}_{A}+\mathbf{u}_{B}\right)
\end{aligned}
$$

\subsection{Mixed mode fracture energy}

We define the mode ratio $\alpha$ as:

$\alpha=\frac{\left(t_{s}^{\text {eff }}\right)^{2}}{\left(t_{s}^{\text {eff }}\right)^{2}+\left\langle t_{n}^{\text {eff }}\right\rangle^{2}}$

This expression can be rewritten to

$\left\langle t_{n}^{\mathrm{eff}}\right\rangle=\left|t_{s}^{\mathrm{eff}}\right| \sqrt{\frac{1-\alpha}{\alpha}}$

Assuming equilibrium between the cohesive traction acting on the crack surface and the bulk stress directly next to this surface $(\mathbf{H} \boldsymbol{\sigma}=\mathbf{t})$, it follows from Eq. 27 that the ratio between the displacement jump components is necessarily the same:

$\llbracket u \rrbracket_{n}=\left|\llbracket u \rrbracket_{s}\right| \sqrt{\frac{1-\alpha}{\alpha}}$

Therefore, for a fixed mode ratio $\alpha$, the ratio between the energy release of the two modes is fixed:

$\frac{G_{I}}{G_{I I}}=\frac{\int t_{n} \mathrm{~d} \llbracket u \rrbracket_{n}}{\int t_{S} \mathrm{~d} \llbracket u \rrbracket_{s}}=\frac{1-\alpha}{\alpha}$

and, consequently

$\frac{G_{I I}}{G_{I}+G_{I I}}=\alpha$

Substitution of Eq. 33 and $\kappa=1$ in Eq. 28 gives the relation between the initial shear traction, $t_{s}^{0}$, and $\alpha$ (knowing that, initially, $\mathbf{t}^{\text {eff }}=\mathbf{t}$ ), which may be written as:

$\left|t_{s}^{0}\right|=\left(\frac{1-\alpha}{\alpha} \cdot \frac{1}{F_{2 \mathrm{t}}^{2}}+\frac{1}{F_{12}^{2}}\right)^{-\frac{1}{2}}=\sqrt{\alpha F_{\alpha}^{2}}$

with

$F_{\alpha}^{2}=\frac{F_{2 \mathrm{t}}^{2} F_{12}^{2}}{(1-\alpha) F_{2 \mathrm{t}}^{2}+\alpha F_{12}^{2}}$ 
Fig. 6 Schematic representation of cohesive law for pure mode I failure (cf. Moonen et al. 2008)
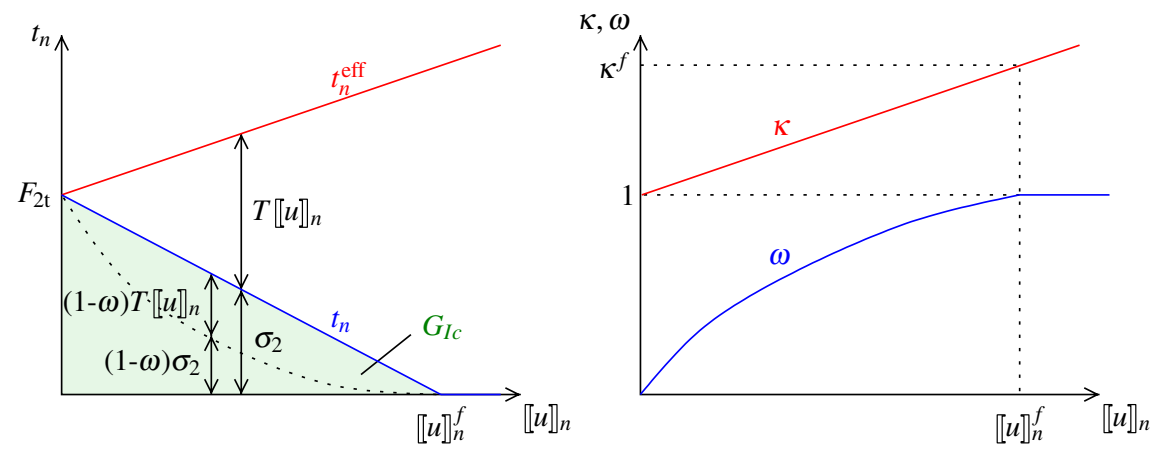

Similarly, substitution of (34) in (28) with $\kappa=\kappa^{f}$ gives the relation between the shear crack opening that corresponds with complete failure, $\llbracket u \rrbracket_{s}^{f}$, and $\alpha$ :

$\left|\llbracket u \rrbracket_{s}^{f}\right|=\frac{\kappa^{f}}{T} \sqrt{\alpha F_{\alpha}^{2}}$

With Eqs. 37 and 39, the actual energy release in mode II can be computed as a function of $\alpha$ (considering that the sign of $t_{s}^{0}$ will always be equal to that of $\left.\llbracket u \rrbracket_{s}^{f}\right)$ :

$G_{I I}=\frac{1}{2} t_{s}^{0} \llbracket u \rrbracket_{s}^{f}=\frac{\kappa^{f} \alpha F_{\alpha}^{2}}{2 T}$

Finally, with Eq. 36, the total energy release as a function of $\alpha$ is:

$G_{T}=G_{I}+G_{I I}=\frac{\kappa^{f} F_{\alpha}^{2}}{2 T}$

Now, $\kappa^{f}$ can be defined as a function of $\alpha$ such that the amount of energy dissipated for different mixed mode cases matches the actual material behavior. We assume that the fracture energy of the material can be described properly with the phenomenological relation proposed for delamination by Benzeggagh and Kenane (1996), which has been applied in interface elements by Camanho et al. (2003) and Turon et al. (2006):

$G_{T c}=G_{I c}+\left(G_{I I c}-G_{I c}\right) \alpha^{\eta}$

in which $G_{I c}$ is the mode I fracture energy, $G_{I I c}$ is the mode II fracture energy, $\eta$ is an additional material parameter, and $\alpha=G_{I I} /\left(G_{I}+G_{I I}\right)$. Equalizing $G_{T c}$ in (42) to $G_{T}$ in (41) and solving for $\kappa^{f}$ gives: $\kappa^{f}=\frac{2 T}{F_{\alpha}^{2}}\left(G_{I c}+\left(G_{I I c}-G_{I c}\right) \alpha^{\eta}\right)$

Because equilibrium was assumed for this derivation, while equilibrium is only weakly met, the amount of energy dissipated in mixed mode conditions does not satisfy Eq. 42 exactly, but it approaches the correct value upon mesh refinement.

Now that the formulation is complete, we comment on the significance of the stiffness parameter $T$. Figure 6 gives a 1D representation of the evolution of different variables with increasing displacement jump, assuming that equilibrium is satisfied exactly. The straight descending line in the left diagram depicts the traction (and at the same time the bulk stress, since those two are in equilibrium). This line is completely defined with the material parameters $F_{2 \mathrm{t}}$ and $G_{I c}$. The increasing straight line in the left diagram is the effective traction. The significance of $T$ is that it determines the slope of this line. As a consequence, it influences the ratio between the 'displacement jump part' and the 'bulk stress part' of the traction (indicated with the curved dotted line), and therewith the influence of substituting $\mathbf{H} \boldsymbol{\sigma}$ for $\mathbf{t}$ in Eq. 23. In the limit for $T \rightarrow \infty$, the direct traction separation law from Eq. 19 is obtained exactly. While, with a finite value for $T$, it is only weakly met. A lower bound for $T$ is given by the fact that $\mathbf{t}^{\mathrm{eff}}$ must be growing during crack opening, i.e., that $\kappa^{f}>1$, which gives with Eq. 43:

$T>\max _{\alpha \in[0,1]} \frac{F_{\alpha}^{2}}{G_{T c}(\alpha)}$

Furthermore, the right diagram in Fig. 6 shows the evolution of $\omega$ from 0 to 1 and of $\kappa$ from 1 to $\kappa^{f}$, the growth of the latter being proportional to the that of $t_{n}^{\text {eff }}$. 


\subsection{Linearization}

For robust analysis of nonlinear problems, consistent linearization of the internal force vector is important. In evaluation of the consistent tangent matrix, the interdependence between a pair of overlapping elements due to the cohesive traction must be taken into account. The consistent tangent matrix for the two overlapping elements together is derived from Eqs. 15 and 16. The contribution related to the bulk stress is standard:

$$
\mathbf{K}_{I}^{\text {bulk }}=\frac{\partial \mathbf{f}_{I}^{\text {bulk }}}{\partial \mathbf{u}_{I}}=\int_{\Omega_{I}} \mathbf{B}^{T} \mathbf{D B} \mathrm{d} \Omega, \quad I=A, B
$$

The contribution related to the cohesive traction has to be derived from the introduced constitutive law.

$$
\frac{\partial \mathbf{f}_{A}^{\mathrm{coh}}}{\partial \mathbf{u}_{I}}=-\frac{\partial \mathbf{f}_{B}^{\mathrm{coh}}}{\partial \mathbf{u}_{I}}=\int_{\Gamma_{c}} \mathbf{N}^{T} \frac{\partial \mathbf{t}}{\partial \mathbf{t}^{\mathrm{eff}}} \frac{\partial \mathbf{t}^{\mathrm{eff}}}{\partial \mathbf{u}_{I}} \mathrm{~d} \Gamma, \quad I=A, B
$$

From the definition of the effective traction (24) it follows

$$
\frac{\partial \mathbf{t}^{\mathrm{eff}}}{\partial \mathbf{u}_{I}}=\mathbf{Q H} \frac{\partial \boldsymbol{\sigma}}{\partial \mathbf{u}_{I}}+T \mathbf{Q} \frac{\partial \llbracket \mathbf{u} \rrbracket}{\partial \mathbf{u}_{I}}, \quad I=A, B
$$

where the kinematic relations (5) and (31) give

$$
\frac{\partial \llbracket \mathbf{u} \rrbracket}{\partial \mathbf{u}_{A}}=-\frac{\partial \llbracket \mathbf{u} \rrbracket}{\partial \mathbf{u}_{B}}=\mathbf{N}
$$

and (with Eq. 10)

$$
\frac{\partial \boldsymbol{\sigma}}{\partial \mathbf{u}_{A}}=\frac{\partial \boldsymbol{\sigma}}{\partial \mathbf{u}_{B}}=\frac{1}{2} \mathbf{D B}
$$

The nonlinearity of the system is in the term $\partial \mathbf{t} / \partial \mathbf{t}^{\text {eff }}$. Considering $\omega$ to be computed from $\mathbf{t}^{\text {eff }}$, we write

$$
\frac{\partial \mathbf{t}}{\partial \mathbf{t}^{\mathrm{eff}}}=\mathbf{Q}^{T} \mathbf{A}
$$

with

$$
\mathbf{A}=\frac{\partial \overline{\mathbf{t}}}{\partial \mathbf{t}^{\mathbf{e f f}}}=\mathbf{I}-\boldsymbol{\Omega}-\overline{\mathbf{t}} \otimes \frac{\partial \omega}{\partial \mathbf{t}^{\mathbf{e f f}}}
$$

In an integration point that is not damaging $(\dot{\omega}=0)$, the final term in Eq. 51 is equal to zero, otherwise it is computed with the following expressions, derived from the definitions for $\omega, \kappa, \kappa^{f}$ and $\alpha$ in Eqs. 29, 28, 43 and 32:

$$
\frac{\partial \omega}{\partial \mathbf{t}^{\mathrm{eff}}}=\frac{\partial \omega}{\partial \kappa} \frac{\partial \kappa}{\partial \mathbf{t}^{\mathrm{eff}}}+\frac{\partial \omega}{\partial \kappa^{f}} \frac{\partial \kappa^{f}}{\partial \alpha} \frac{\partial \alpha}{\partial \mathbf{t}^{\mathrm{eff}}}, \quad j=n, s
$$

with

$$
\begin{aligned}
\frac{\partial \omega}{\partial \kappa} & =\frac{\kappa^{f}}{\kappa^{2}\left(\kappa^{f}-1\right)} \\
\frac{\partial \omega}{\partial \kappa^{f}} & =\frac{1-\kappa}{\kappa\left(\kappa^{f}-1\right)^{2}} \\
\frac{\partial \kappa^{f}}{\partial \alpha} & =\frac{2 T}{F_{\alpha}^{2}} \frac{\partial G_{T c}}{\partial \alpha}-\frac{2 T G_{T c}}{F_{\alpha}^{4}} \frac{\partial F_{\alpha}^{2}}{\partial \alpha} \\
\frac{\partial \kappa}{\partial \mathbf{t}^{\mathrm{eff}}} & =\left\{\frac{\left\langle t_{n}^{\mathrm{eff}}\right\rangle}{\kappa F_{2 \mathrm{t}}^{2}} ; \frac{t_{s}^{\mathrm{eff}}}{\kappa F_{12}^{2}}\right\}^{T} \\
\frac{\partial \alpha}{\partial \mathbf{t}^{\mathrm{eff}}} & =\left\{\frac{2 \alpha(1-\alpha)}{t_{s}^{\mathrm{eff}}} ;-\frac{2 \alpha(1-\alpha)}{t_{n}^{\mathrm{eff}}}\right\}^{T}
\end{aligned}
$$

in which

$$
\begin{aligned}
\frac{\partial G_{T c}}{\partial \alpha} & =\left(G_{I I c}-G_{I c}\right) \eta \alpha^{\eta-1} \\
\frac{\partial F_{\alpha}^{2}}{\partial \alpha} & =\frac{F_{2 \mathrm{t}}^{2} F_{12}^{2}\left(F_{2 \mathrm{t}}^{2}-F_{12}^{2}\right)}{\left((1-\alpha) F_{2 \mathrm{t}}^{2}+\alpha F_{12}^{2}\right)^{2}}
\end{aligned}
$$

The consistent tangent matrix for two overlapping elements together can be summarized as

$$
\begin{aligned}
& \mathbf{K}=\left[\begin{array}{cc}
\mathbf{K}_{A}^{\text {bulk }} & 0 \\
0 & \mathbf{K}_{B}^{\text {bulk }}
\end{array}\right]+\left[\begin{array}{cc}
\mathbf{K}_{\llbracket u \rrbracket}^{\text {coh }} & -\mathbf{K}_{\llbracket u \rrbracket}^{\mathrm{coh}} \\
-\mathbf{K}_{\llbracket u \rrbracket}^{\text {coh }} & \mathbf{K}_{\llbracket u \rrbracket}^{\text {coh }}
\end{array}\right] \\
&+\left[\begin{array}{cc}
\mathbf{K}_{\sigma}^{\mathrm{coh}} & \mathbf{K}_{\sigma}^{\mathrm{coh}} \\
-\mathbf{K}_{\sigma}^{\mathrm{coh}} & -\mathbf{K}_{\sigma}^{\mathrm{coh}}
\end{array}\right]
\end{aligned}
$$

with $\mathbf{K}_{I}^{\text {bulk }}$ from Eq. 45 and

$$
\begin{aligned}
& \mathbf{K}_{\llbracket u \rrbracket}^{\mathrm{coh}}=T \int_{\Gamma_{c}} \mathbf{N}^{T} \mathbf{Q}^{T} \mathbf{A} \mathbf{Q N} \mathrm{d} \Gamma \\
& \mathbf{K}_{\sigma}^{\mathrm{coh}}=\frac{1}{2} \int_{\Gamma_{c}} \mathbf{N}^{T} \mathbf{Q}^{T} \mathbf{A} \mathbf{Q H D B} \mathrm{d} \Gamma
\end{aligned}
$$

\section{Loading strategy}

When splitting occurs in fibrous materials, whether or not in combination with delamination, two processes take place simultaneously:

- matrix material damages (by definition), and

- fibers unload (as a possible consequence).

Because the fibers are very stiff and the matrix failure process is not very ductile, the amount of elastic energy released by the second process easily exceeds the amount of energy necessary to drive the first. Hence, unstable crack propagation occurs, and snapback behavior is observed when the equilibrium path is followed. 
In the examples in this paper, a dissipation-based arclength method is employed to follow this behavior. For details of this method we refer to the work by Gutiérrez (2004) and Verhoosel et al. (2008). As usual in arclength methods, a constraint equation is added to the system of equations that is solved iteratively. In this particular formulation, the constraint equation is defined such that it is only satisfied when a predefined amount of energy is dissipated in the time step.

The dissipation-based arclength method is only applicable when the material is damaging. Therefore it is not suitable for the initial phase of the analysis or for possible elastic loading stages which may occur after a snapback. Therefore, a hybrid loading strategy is adopted in which load control is used in some of the time steps and arclength control in the other time steps.

The analysis is started in load control. For every converged solution the amount of dissipated energy is computed, also when the converged solution does not satisfy the failure criterion (see Fig. 4). When this amount exceeds a certain threshold value, we switch to arclength control and this amount is used as initial value for the prescribed energy dissipation.

As explained in Sect. 2.3, the increment is reduced, when the Newton-Raphson procedure does not converge, where the increment is either the prescribed amount of dissipated energy or the load increment. However, when this increment becomes too small, we switch from the current strategy to the other. Only when, within a given time step, no converged solution can be found in a range of increments for both loading strategies, is the computation terminated. Also when in the arclength method the factor with which the external force vector is scaled changes sign, which may happen when the load approaches zero during brittle snapback, a switch to load control is made.

\section{Numerical Examples}

\subsection{Mixed mode bending}

The performance of the cohesive law is assessed with mixed mode bending tests. Material parameters from Camanho et al. (2003) for carbon/PEEK fiber reinforced composite are used (see Table 1). The response is governed by the fracture energy of the material. Therefore, these tests are used to validate whether the simu-
Table 1 Material parameters for mixed mode bending and crossply laminate example (Camanho et al. 2003)

\begin{tabular}{llll}
\hline Elasticity & \multicolumn{3}{c}{ Splitting } \\
\hline$E_{1}$ & $122.7 \mathrm{GPa}$ & $F_{2 \mathrm{t}}$ & $80 \mathrm{MPa}$ \\
$E_{2}$ & $10.1 \mathrm{GPa}$ & $F_{12}$ & $100 \mathrm{MPa}$ \\
$\nu_{12}$ & 0.25 & $G_{I c}$ & $0.969 \mathrm{~N} / \mathrm{mm}$ \\
$G_{12}$ & $5.5 \mathrm{GPa}$ & $G_{I I c}$ & $1.719 \mathrm{~N} / \mathrm{mm}$ \\
& & $\eta$ & 2.284 \\
\hline
\end{tabular}

lated response matches the theoretical response for the input fracture energy in pure and mixed mode cases. For an optimal match with theoretical solutions derived from beam theory, plane stress analysis is considered to be most appropriate.

The setup for the mixed mode bending (MMB) test (see Fig. 7) can be used with minor modifications for the pure mode cases of a double cantilever beam (DCB) and end notch flexure (ENF). The mode I DCB test is obtained by setting $F_{m}$ equal to zero, and the mode II ENF test is obtained by setting $F_{e}$ equal to zero or replacing the end force with a nodal displacement constraint. Theoretical solutions exist for all mixed mode bending tests, derived from theoretical solutions for the extreme cases. With beam theory and the assumption that the fracture energy is constant, the force and displacement during crack propagation for each value of crack length $a$ can be obtained. We have used relations concatenated by Reeder and Crews (1990) composed from the work of Kanninen (1973); Whitney et al. (1982) and Carlsson et al. (1986).

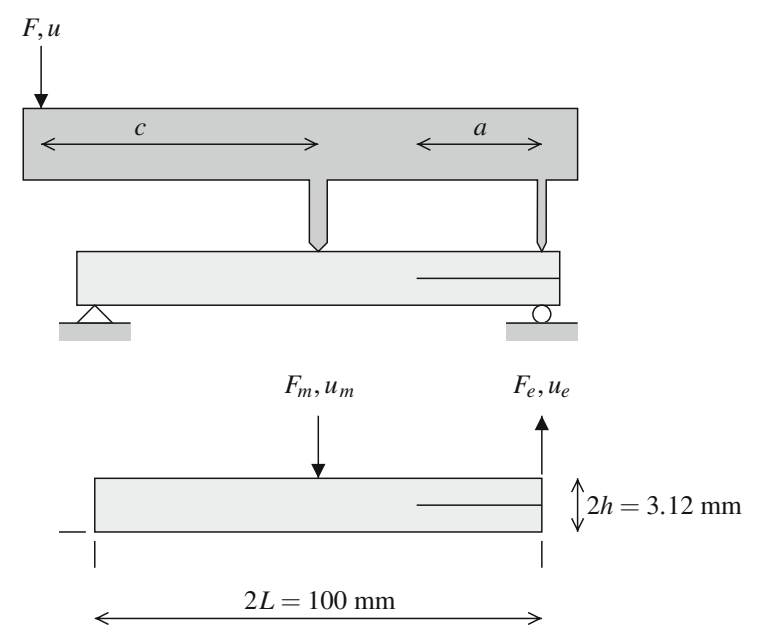

Fig. 7 Mixed mode bending test setup (top) and computational model (bottom) 
The theoretical solutions to the pure mode cases of the DCB and ENF tests will be presented first, followed by the theoretical solution for the MMB tests.

For the DCB test, the following relations are used. These include shear deformation (Whitney et al. 1982) and the deformation of the uncracked part of the specimen derived from analogy with a beam on elastic foundation (Kanninen 1973). The fracture energy is related to the load with

$G_{T}=G_{I}=\frac{12 F_{e}^{2} a^{2}}{E_{1} b^{2} h^{3}}\left(1+\frac{2 h}{\gamma a}+\frac{h^{2}}{\gamma^{2} a^{2}}+\frac{E_{1} h^{2}}{10 G_{12} a^{2}}\right)$

where $b$ is the width of the beam, in this paper the unit width, and

$\gamma=\sqrt[4]{\frac{6 E_{2}}{E_{1}}}$

The terms in Eq. 63 with $\gamma$ are related to elastic deformation of the uncracked part and the term with $G_{12}$ is related to shear deformation. The first of these terms is most significant. With Eq. 63, the force corresponding with a certain fracture energy $G_{c}=G_{I}$ and crack length $a$ is computed. Subsequently, the corresponding displacement $u_{e}$ is computed with:

$u_{e}=\frac{8 F_{e} a^{3}}{E_{1} b h^{3}}\left(1+\frac{3 h}{\gamma a}+\frac{3 h^{2}}{\gamma^{2} a^{2}}+\frac{3 h^{3}}{4 \gamma^{3} a^{3}}+\frac{3 E_{1} h^{2}}{8 G_{12} a^{2}}\right)$

For the ENF test, the following relations, including shear deformation (Carlsson et al. 1986), are used

$G_{T}=G_{I I}=\frac{9 F_{m}^{2} a^{2}}{16 E_{1} b^{2} h^{3}}\left(1+\frac{E_{1} h^{2}}{5 G_{12} a^{2}}\right)$

and

$$
\begin{aligned}
u_{m}= & \frac{F_{m}\left(2 L^{3}+3 a^{3}\right)}{8 E_{1} b h^{3}} \\
& \left(1+\frac{(2.4 L+1.8 a) E_{1} h^{2}}{\left(2 L^{3}+3 a^{3}\right) G_{12}}\right)
\end{aligned}
$$

For the MMB tests, the theoretical solutions from both pure mode cases are combined. With $F$ as the force acting at a distance $c$ from the middle of the specimen (see Fig. 7), equilibrium considerations give the middle and end loads as:

$$
\begin{aligned}
F_{m} & =\frac{c+L}{L} F \\
F_{e} & =\frac{c}{L} F
\end{aligned}
$$

The pure mode forces are related to these, but not exactly equal, because when there is a force $F_{m}$ acting at the middle, part of the force $F_{e}$ acting at the end is acting as reaction force. Therefore, the pure mode load components are defined as:

$$
\begin{aligned}
F_{I} & =F_{e}-\frac{1}{4} F_{m}=\frac{3 c-L}{4 L} F \\
F_{I I} & =F_{m}=\frac{c+L}{L} F
\end{aligned}
$$

The total fracture energy in mixed mode loading is obtained using Eqs. 63 and 66:

$$
\begin{aligned}
G_{T}= & G_{I}+G_{I I} \\
= & \frac{12 F_{I}^{2} a^{2}}{E_{1} b^{2} h^{3}}\left(1+\frac{2 h}{\gamma a}+\frac{h^{2}}{\gamma^{2} a^{2}}+\frac{E_{1} h^{2}}{10 G_{12} a^{2}}\right) \\
& \quad+\frac{9 F_{I I}^{2} a^{2}}{16 E_{1} b^{2} h^{3}}\left(1+\frac{E_{1} h^{2}}{5 G_{12} a^{2}}\right)
\end{aligned}
$$

and

$$
\begin{aligned}
u= & \frac{L+c}{L} u_{m}+\frac{c}{L} u_{e}=\frac{L+c}{L} \frac{F_{I I}\left(2 L^{3}+3 a^{3}\right)}{8 E_{1} b h^{3}} \\
& \times\left(1+\frac{(2.4 L+1.8 a) E_{1} h^{2}}{\left(2 L^{3}+3 a^{3}\right) G_{12}}\right)+\frac{c}{L} \frac{8 F_{I} a^{3}}{E_{1} b h^{3}} \\
& \times\left(1+\frac{3 h}{\gamma a}+\frac{3 h^{2}}{\gamma^{2} a^{2}}+\frac{3 h^{3}}{4 \gamma^{3} a^{3}}+\frac{3 E_{1} h^{2}}{8 G_{12} a^{2}}\right) .
\end{aligned}
$$

In the numerical model, the ratio between the two point loads $F_{m}$ and $F_{e}$ was fixed directly, so the loading arm was not modeled. The relation between load ratio and theoretical arm length $c$ is:

$\frac{F_{e}}{F_{m}}=\frac{c}{c+L}$

The values for the arm length that correspond with the desired values for the mode ratio were computed numerically by substituting different values of $c$ in the relations given above and computing the ratio $\alpha$ corresponding with these values. Due to the correction terms for shear deformation and elastic deformation of the uncracked part, the mode ratio does not depend purely on the arm length $c$ but also on the crack length $a$. However, the influence of $a$ remains limited. The applied values for $c$ are: 98.5, 42.5, and $27.7 \mathrm{~mm}$ for $\alpha$ equals $0.2,0.5$, and 0.8 , respectively. These correspond with $a=30 \mathrm{~mm}$, which is the initial crack length. The influence of crack propagation on the mode ratio is such that with these load ratios, when $a$ increases to $45 \mathrm{~mm}$ (beyond which the crack does not grow in the presented results) the theoretical values for 


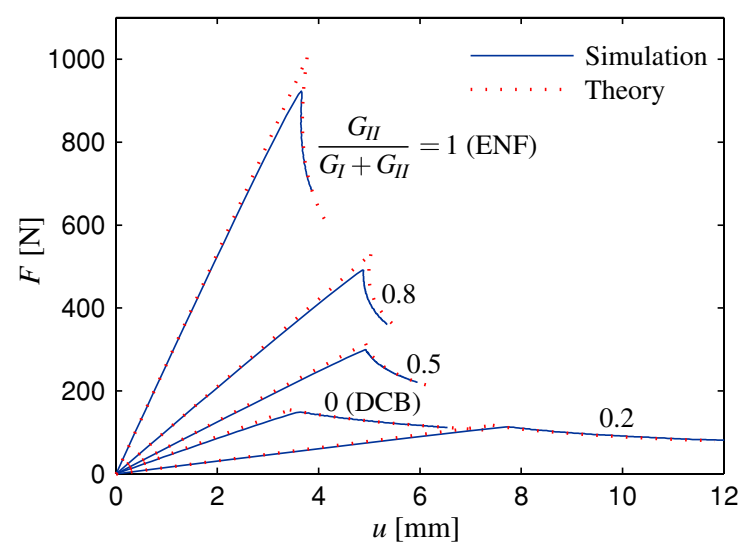

Fig. 8 Load displacement relation for DCB, MMB and ENF tests, compared with theory

the mode ratio increase slightly to $0.206,0.509$ and 0.806 .

Figure 8 shows the relations between load and displacement obtained with the presented model for the two pure mode and three mixed mode cases. Theoretical curves are also shown. For the mixed mode cases, the load on the $\operatorname{arm} F$ is plotted against the deflection of the loading point $u$, while for the pure mode cases it is $F_{e}$ against $u_{e}$ and $F_{m}$ against $u_{m}$. It can be observed that agreement is excellent, at least as far as the initial stiffness and the descending branch are concerned. The agreement in peak load value is not that well. This can be explained by the fact that in the theoretical solution, there is no cohesive zone, in contrast with the numerical model. In the ascending branch, there is already some crack propagation in the numerical model. The peak load corresponds with the moment at which complete local failure is reached for the first time, i.e., when the cohesive zone has fully developed. At that point, there has already been some loss in stiffness, which can be interpreted as an increase of the effective crack length. For more brittle cases, e.g., with a larger specimen or a lower fracture energy, this difference will vanish.

Figure 9 visualizes the traction separation relation as it is obtained for $\alpha=0.5$. For a single cohesive integration point, the evolution of both components of the traction is presented. Notably, the mode ratio is not constant in the cohesive zone. Initially, the traction is predominantly shear traction, and during the crack opening the normal component becomes more important. In the derivation used to incorporate the fracture

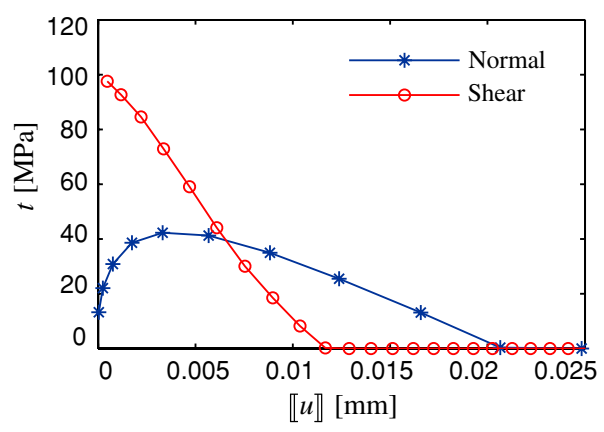

Fig. 9 Evolution of traction and displacement jump components in a single integration point for $\alpha=0.5$

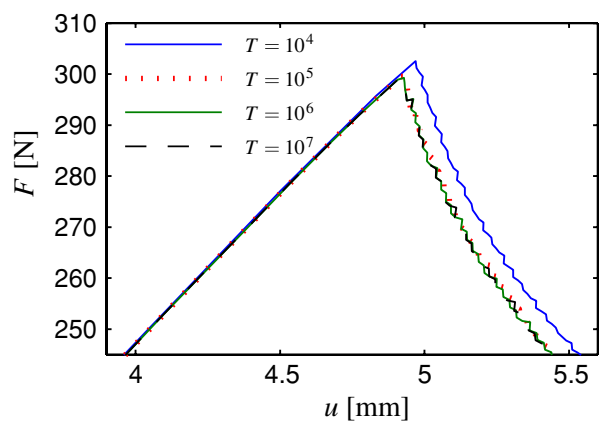

Fig. 10 Zoom of the load displacement data for the MMB test with $\alpha=0.5$ for different values of $T$ (in $\mathrm{N} / \mathrm{mm}^{3}$ )

energy law in the cohesive model, it was assumed that the mode ratio is constant. As a consequence, the fracture energy is initially overpredicted in each integration point (since $G_{I I c}>G_{I c}$ ), and later underpredicted. This may very well be the cause for the fact that the agreement between theory and simulation is slightly less favorable for the mixed mode cases than for the pure mode cases (see Fig. 8). However, from the generally good agreement, it can be concluded that the initial overprediction is balanced well by the subsequent underprediction.

Furthermore, the sensitivity to changes in the parameter $T$ was investigated. Since this is merely a numerical parameter, it should not influence the response. Figure 10 shows a close-up of the load displacement relation for the MMB test with $\alpha=0.5$, using a range of values for $T$. It can be observed that a relatively low value of $T$ gives rise to a small deviation in the results, but for sufficiently high $T$ further changes do not affect the results. Convergence was optimal for $T \in\left[10^{5}, 10^{6}\right]$. All other results presented in this paper, were obtained using $T=10^{5} \mathrm{~N} / \mathrm{mm}^{3}$. 


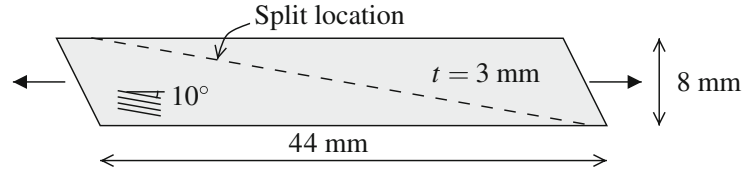

Fig. 11 Off-axis tensile test dimensions

Table 2 Material parameters for off-axis tensile test (Van Paepegem et al. 2006)

\begin{tabular}{llll}
\hline Elasticity & \multicolumn{3}{l}{ Splitting } \\
\hline$E_{1}$ & $38.9 \mathrm{GPa}$ & $F_{2 \mathrm{t}}$ & $36.5 \mathrm{MPa}$ \\
$E_{2}$ & $13.3 \mathrm{GPa}$ & $F_{12}$ & $52 \mathrm{MPa}$ \\
$\nu_{12}$ & 0.26 & $G_{I c}$ & $3 \mathrm{~N} / \mathrm{mm}$ \\
$G_{12}$ & $5.13 \mathrm{GPa}$ & $G_{I I c}$ & $3 \mathrm{~N} / \mathrm{mm}$ \\
& & $\eta$ & 1 \\
\hline
\end{tabular}

\subsection{Off-axis tensile test}

Next, we consider an off-axis tensile test on a $10^{\circ}$ unidirectional laminate. This is a standard test for the determination of the in-plane shear strength (Chamis and Sinclair 1977; Van Paepegem et al. 2006). Experiments show brittle matrix failure. In a sudden event, the specimen breaks, with the crack running in fiber direction, as shown in Fig. 11. In this relatively simple case, regularized continuum models tend to fail in capturing the mechanism correctly (van der Meer and Sluys 2009). Material parameters for glass/epoxy from Van Paepegem et al. (2006) are used (see Table 2).

Notably, the geometry is such, that the stress field is homogeneous until nonlinearities occur, which means that the failure criterion is exactly satisfied in the entire specimen when the crack is initiated. Therefore, the location where the crack initiates is specified in advance.

In Fig. 12, the load displacement relation is plotted for two different meshes, one with an average element size $1.1 \times 0.5 \mathrm{~mm}$ and the other with average element size $0.8 \times 0.25 \mathrm{~mm}$. It can be observed that with the discontinuous approach mesh-independent results are obtained without special precautions, which stands in contrast to local continuum models for failure.

Figure 13 shows the deformed mesh from the fine mesh solution for different stages. The normalized stress quantity that is used for the shading is the failure criterion in Eq. 17. In Fig. 12, the corresponding points on the load displacement curve are indicated. In a sudden event at the peak load level, a cohesive crack of con-

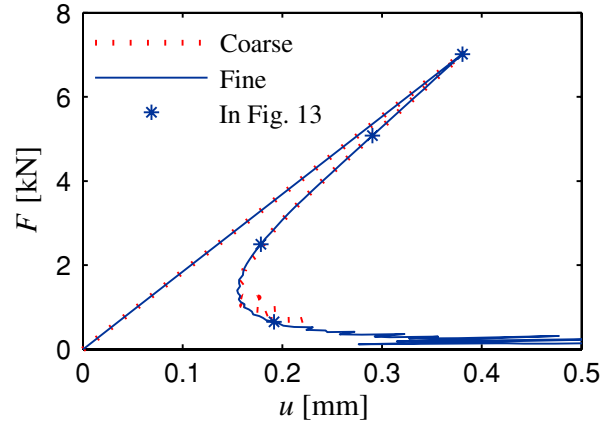

Fig. 12 Load displacement data for off-axis test with phantom node method
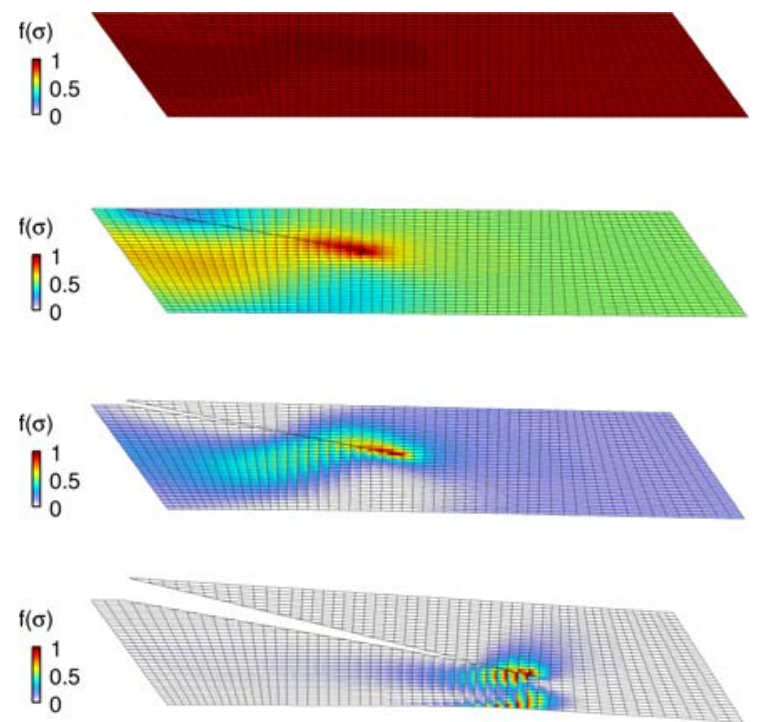

Fig. 13 Off-axis tensile test results with phantom node method (fine mesh, unscaled deformations)

siderable length appears, after which the load drops in a sharp snapback without much additional crack growth. Then the cohesive zone, i.e., the part of the crack in which tractions are present, becomes smaller and the crack finds its way toward the opposite boundary of the specimen. The crack grows in the correct direction. This is in itself not surprising, since the growth direction is predefined, but it is exactly this, that the direction of crack propagation can be fixed, which should be considered an advantage of the discontinuous approach.

\subsection{Cross-ply laminate with a hole}

Finally, it is demonstrated how the model for splitting of a single ply can be applied in laminate analysis. 
For that purpose, it is necessary to capture the interaction between splitting and delamination. A $[ \pm 45]_{\mathrm{s}^{-}}$ laminate with a circular hole loaded in tension is analyzed (see Fig. 14). Because the loading and the main failure mechanism for this case are typically in-plane, a plane-stress approach is adopted (cf. Wisnom and Chang 2000), i.e., each ply is represented as a 2D plane, and the plies are connected with plane interface elements with a shear damage law. Thus, mode I delamination is not considered, but on the other hand, the number of degrees of freedom is reduced with a factor 3 in comparison with a 3D approach with a single layer of volume elements or solid-like shell elements per ply.

Two plies are modeled, which should be understood as half of a symmetric laminate in which case the absence of torsion is justified. The ply thickness is $0.2 \mathrm{~mm}$.

A simple damage law is assigned to the interface elements. The model has two independent parameters: strength $t^{\max }$ and fracture energy $G_{c}$, and a third parameter, which is derived from the elasticity parameters, viz. elastic stiffness $K$ (see Fig. 15 and Table 3). The

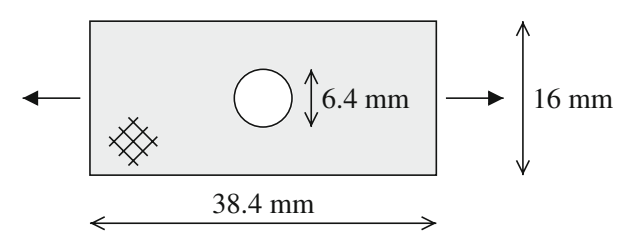

Fig. 14 Cross-ply laminate with a circular hole loaded in tension

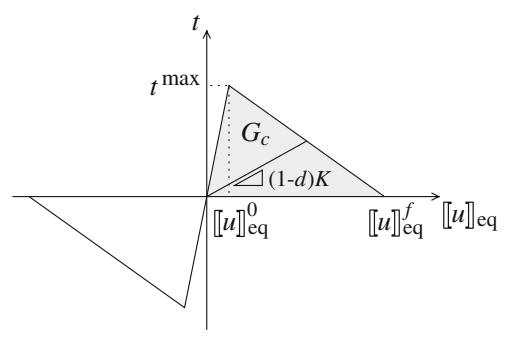

Fig. 15 Constitutive law for interface elements

Table 3 Additional parameters for cross-ply laminate example (see Table 1 for other material parameters)

\section{Delamination}

\begin{tabular}{ll}
\hline$t^{\max }$ & $45 \mathrm{MPa}$ \\
$G_{c}$ & $0.5 \mathrm{~N} / \mathrm{mm}$ \\
$K$ & $27,500 \mathrm{~N} / \mathrm{mm}^{3}$ \\
\hline
\end{tabular}

traction is computed with:

$$
\llbracket u \rrbracket_{\mathrm{eq}}=\sqrt{\llbracket u \rrbracket_{x}^{2}+\llbracket u \rrbracket_{y}^{2}}
$$

$d=\max _{t \leq \tau} \begin{cases}0, & \llbracket u \rrbracket_{\mathrm{eq}} \leq \llbracket u \rrbracket_{\mathrm{eq}}^{0} \\ \frac{\left(\llbracket u \rrbracket_{\mathrm{eq}}-\llbracket u \rrbracket_{\mathrm{eq}}^{0}\right) \llbracket u \rrbracket_{\mathrm{eq}}^{f}}{\llbracket u \rrbracket_{\mathrm{eq}}\left(\llbracket u \rrbracket_{\mathrm{eq}}^{f}-\llbracket u \rrbracket_{\mathrm{eq}}^{0}\right)}, & \llbracket u \rrbracket_{\mathrm{eq}}^{0}<\llbracket u \rrbracket_{\mathrm{eq}}<\llbracket u \rrbracket_{\mathrm{eq}}^{f} \\ 1, & \llbracket u \rrbracket_{\mathrm{eq}} \geq \llbracket u \rrbracket_{\mathrm{eq}}^{f}\end{cases}$

$\mathbf{t}=(1-d) K \llbracket \mathbf{u} \rrbracket$

with

$\llbracket u \rrbracket_{\mathrm{eq}}^{0}=\frac{t^{\mathrm{max}}}{K}$

$\llbracket u \rrbracket_{\mathrm{eq}}^{f}=\frac{2 G_{c}}{t^{\max }}$

and

$K=\frac{G_{12}}{\Delta z}$

where $G_{12}$ is the in-plane shear stiffness $\Delta z$ is the ply thickness. Equation 80 is derived from the idea that the mid-planes of the ply can move with respect to one another due to out-of-plane shear. Ideally, the stiffness would have to be orthotropic and dependent on the fiber direction in the plies on both sides of the interface, but since the difference between $G_{31}$ and $G_{23}$ is generally not very big and the value of $K$ is not of key importance, we stick to this simple dependence on $G_{12}$, a parameter that is already required for the ply model.

Cracks are allowed to initiate anywhere along the boundary or in the interior of the ply, with only one restriction, that the projected spacing between two cracks in the same ply is at least $0.7 \mathrm{~mm}$. This distance is related to the typical element size. The resolution of the crack pattern that can be captured is related to the fineness of the discretization. At least one intact element should be located between two elements that are cut by two different cracks. When the failure criterion is violated in an element that lies on the path of an existing crack but yet ahead of its tip, a new crack is introduced in this element and its location is such that this new crack will form one straight line with the existing crack when they meet.

Figure 16 shows the peculiar relation between load and displacement that is obtained. Many sharp snapbacks are visible. The nature of these snapbacks can be explained with Figs. 17 and 18, the former showing the 


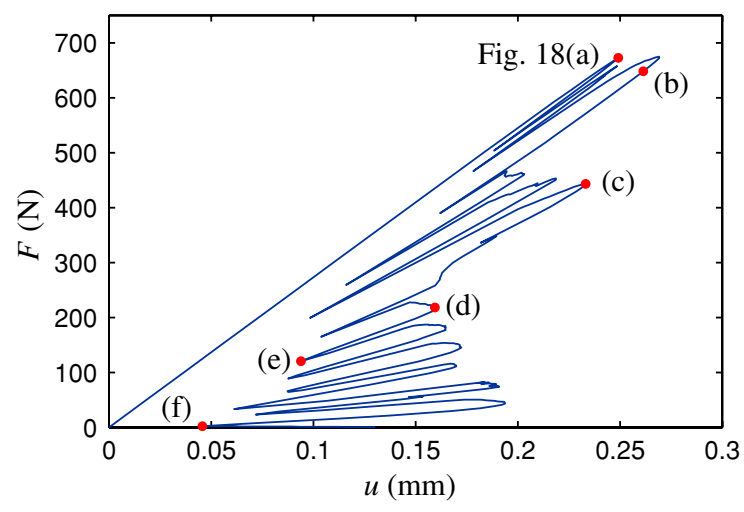

Fig. 16 Load displacement diagram for cross-ply laminated with splitting and delamination. The dots indicate the time steps for which results are shown in Fig. 18

deformed mesh at a late stage and the latter visualizing the evolution of interface damage and splitting cracks. Figure 17 is taken from the same time step as Fig. 18d. At this stage, there is a fiber bundle on both sides of the hole that connects the two halves of the top ply. The snapback occurs when the boundary is reached by one of the splits in these fiber bundles. Then the material on one side of this crack suddenly unloads. During the snapback, the whole specimen is unloaded, and when it is reloaded, the crack that has just reached the boundary is opening, leaving its neighbor that was previously opening, closed. The corresponding additional delamination is visible at the middle of the base of Fig. 18e: the area right of the crack that has reached the boundary has completely delaminated.

Furthermore, when looking at the load displacement response and the damage evolution, several observations can be made. Firstly, that already before the peak significant splitting occurs, as well as initial delamination near the hole (Fig. 18a). The peak is reached when delamination starts along the complete length of one of the four main splits (Fig. 18b). Then delamination propagates first only on one side of the hole, accompanied by distributed cracking in one of the plies (Fig. 18c). Then delamination on the other side of the hole follows, until final failure is obtained (Fig. 18f).

Theoretically, the response for this problem should be symmetric. However, clear asymmetry can be observed in the solution. This asymmetry is initially triggered by asymmetry in the mesh. Furthermore, the algorithm in which cracks propagate one by one, promotes minor asymmetry in the response. And minor asymmetry suffices for the extremely brittle failure events to occur sequentially on both sides of the hole instead of concurrently.

It can be observed that distributed matrix cracking is predicted in part of the specimen and that the obtained crack spacing is governed by the minimum spacing that was given as a numerical parameter. The question how to represent the very fine distributed cracking that may occur remains open, notwithstanding this example shows the capability of the proposed method to simulate failure in laminates when it is driven by ply splitting.

\section{Conclusions}

The phantom node method has been implemented to model progressive splitting in laminates. The phenomenon that a split grows by definition in fiber direction is enforced by setting the direction of crack propagation equal to the fiber direction. Cracks do not have to be meshed. And multiple cracks can be captured, without having to predefine their location.

For robust implicit analysis of mixed mode cohesive cracking, a new cohesive law is applied. The traction acting on the cohesive surface is not computed directly from the displacement jump, but constrained with the bulk stress in the continuum next to it. A linear softening relation between traction and separation is weakly

Fig. 17 Deformed mesh just before a snapback occurs (deformations are magnified with a factor 10 ), cf. Fig. 18d

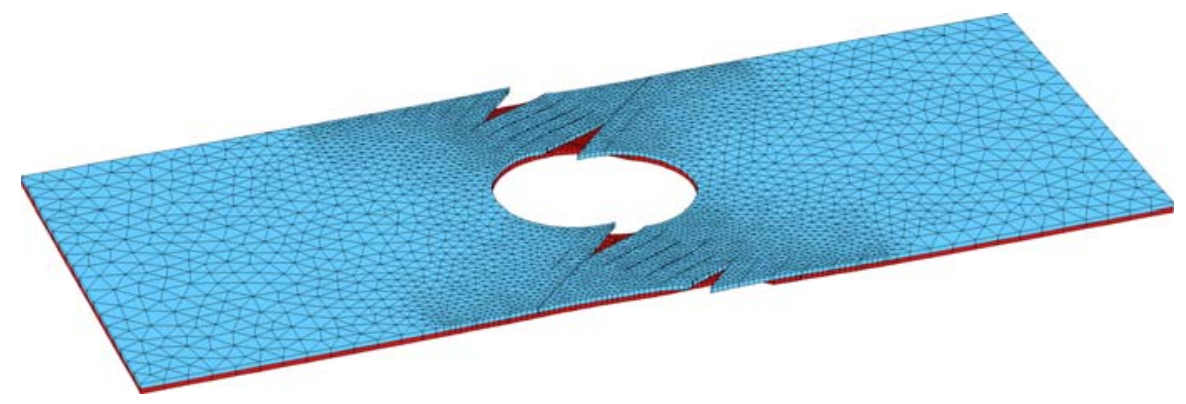


Fig. 18 Delamination damage and cracks for different time steps (see Fig. 16)

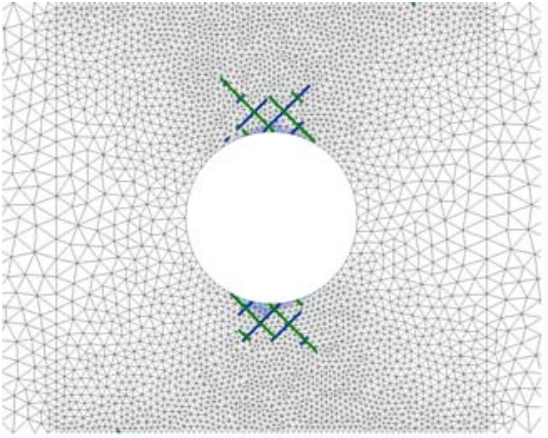

(a)

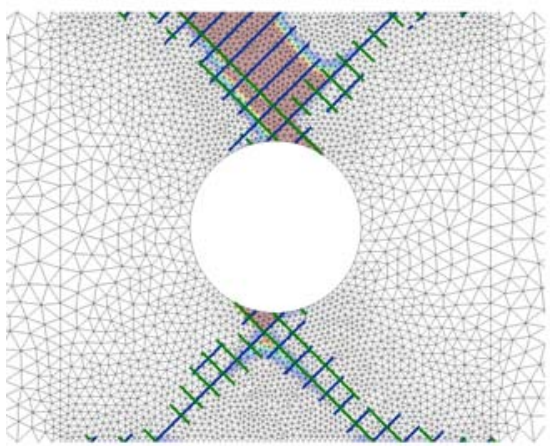

(c)

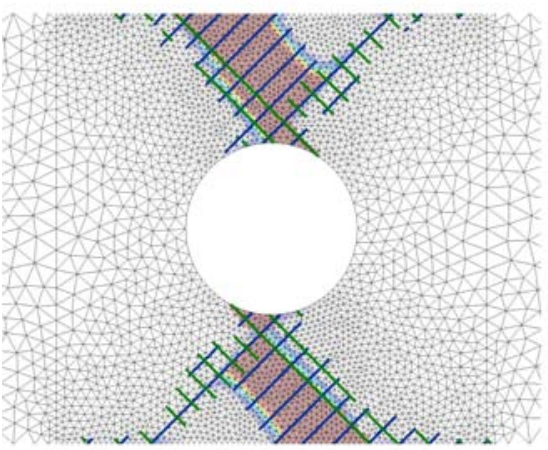

(e)

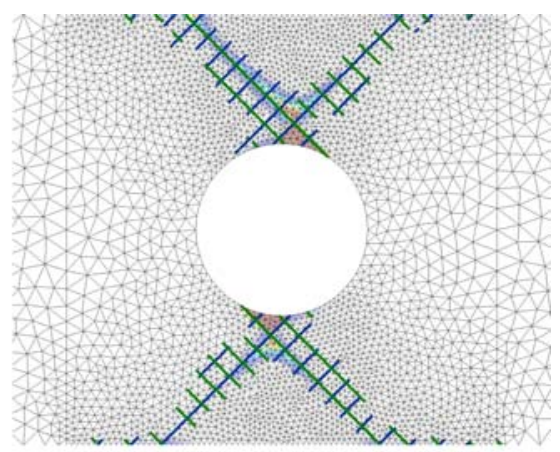

(b)

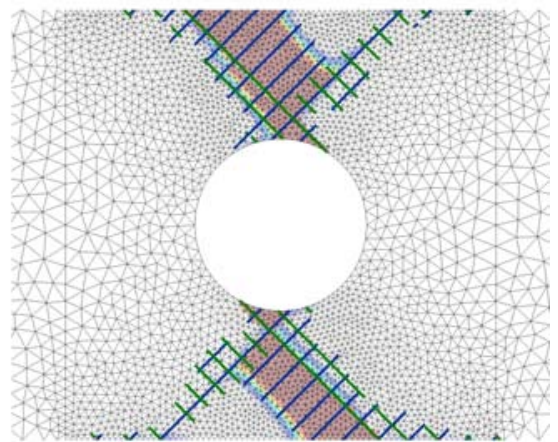

(d)

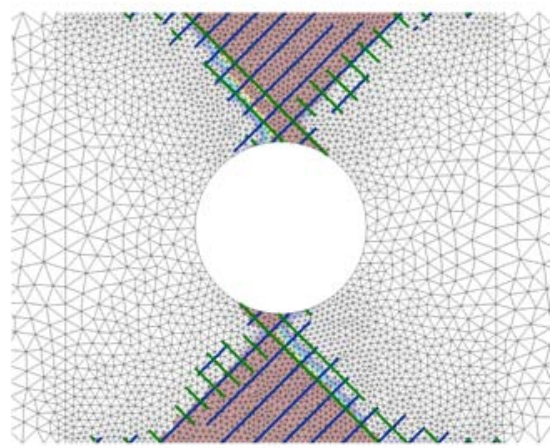

(f)

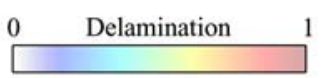

imposed with strength and fracture energy as essential parameters.

The validity of the cohesive law is assessed with a series of simulations of the mixed mode bending test. For this test, which has a response that is typically governed by the fracture energy, results are in agreement with those from theory. It has been shown that the input fracture energy, though only weakly imposed, is indeed obtained accurately for pure mode cases as well as for a range of mixed mode cases.
The $10^{\circ}$ off-axis tensile test was simulated to illustrate the capability of this approach in capturing the typical splitting crack pattern. Regularized continuum models tend to fail in predicting the correct failure pattern for this basic case, while with the phantom node method with fixed crack propagation direction, the correct outcome is obtained.

The intended use of the presented model lies in the analysis of failure in complete laminates, in which case splitting is not the only failure process. To dem- 
onstrate the performance of the presented method in full laminate analysis, splitting and delamination in a notched cross-ply laminate was analyzed in a plane stress framework. A simple cohesive law was applied to interface elements for delamination. Promising results were obtained in which numerous splitting cracks were predicted, some of which eventually interacted with delamination to form the final failure mechanism.

It is envisioned that the model can be combined with a separate description for fiber failure to allow for complete mesoscopic analysis of progressive failure in laminates.

Acknowledgements This research is supported by the Technology Foundation STW (under Grant DCB.6623) and the Ministry of Public Works and Water Management, The Netherlands.

Open Access This article is distributed under the terms of the Creative Commons Attribution Noncommercial License which permits any noncommercial use, distribution, and reproduction in any medium, provided the original author(s) and source are credited.

\section{References}

Belytschko T, Black T (1999) Elastic crack growth in finite elements with minimal remeshing. Int $\mathrm{J}$ Numer Method Eng 45(5):601-620

Benzeggagh ML, Kenane M (1996) Measurement of mixedmode delamination fracture toughness of unidirectional glass/epoxy composites with mixed mode bending apparatus. Compos Sci Technol 56(4):439-449

Camanho PP, Dávila CG, Moura MF (2003) Numerical simulation of mixed-mode progressive delamination in composite materials. J Compos Mater 37(16):1415-1438

de Carlsson LA, Gillespie JW Jr, Pipes RB (1986) On the analysis and design of the end notched flexure (ENF) specimen for mode II testing. J Compos Mater 20(6):594-604

Chamis CC, Sinclair JH (1977) Ten-deg off-axis test for shear properties in fiber composites. Exp Mech 17(9):339-346

Dávila CG, Camanho PP, Rose CA (2005) Failure criteria for FRP laminates. J Compos Mater 39:323-345

Green BG, Wisnom MR, Hallett SR (2007) An experimental investigation into the tensile strength scaling of notched composites. Compos Part A 38(3):867-878

Gutiérrez MA (2004) Energy release control for numerical simulations of failure in quasi-brittle solids. Commun Numer Method Eng 20(1):19-29

Hallett SR, Wisnom MR (2006a) Experimental investigation of progressive damage and the effect of layup in notched tensile tests. J Compos Mater 40(2):119-141

Hallett SR, Wisnom MR (2006b) Numerical investigation of progressive damage and the effect of layup in notched tensile tests. J Compos Mater 40(14):1229-1245

Hansbo A, Hansbo P (2004) A finite element method for the simulation of strong and weak discontinuities in solid mechanics. Comput Method Appl Mech Eng 193(3335):3523-3540

Hashin Z (1980) Failure criteria for unidirectional fiber composites. J Appl Mech 47:329-334

Iarve EV (2003) Mesh independent modelling of cracks by using higher order shape functions. Int $\mathbf{J}$ Numer Method Eng 56(6):869-882

Jiang WG, Hallett SR, Green BG, Wisnom MR (2007) A concise interface constitutive law for analysis of delamination and splitting in composite materials and its application to scaled notched tensile specimens. Int J Numer Method Eng 69(9):1982-1995

Kanninen MF (1973) An augmented double cantilever beam model for studying crack propagation and arrest. Int J Fract 9(1):83-92

Lapczyk I, Hurtado JA (2007) Progressive damage modeling in fiber-reinforced materials. Compos Part A 38(11):23332341

Laurin F, Carrère N, Maire JF (2007) A multiscale progressive failure approach for composite laminates based on thermodynomical viscoelastic and damage models. Compos Part A 38(1):198-209

Maimí P, Camanho PP, Mayugo JA, Dávila CG (2007) A continuum damage model for composite laminates: Part I-constitutive model. Mech Mater 39(10):897-908

Matzenmiller A, Lubliner J, Taylor RL (1995) A constitutive model for anisotropic damage in fiber-composites. Mech Mater 20(2):125-152

Mergheim J, Kuhl E, Steinmann P (2005) A finite element method for the computational modelling of cohesive cracks. Int J Numer Method Eng 63(2):276-289

Moës N, Belytschko T (2002) Extended finite element method for cohesive crack growth. Eng Fract Mech 69(7):813-833

Moës N, Dolbow J, Belytschko T (1999) A finite element method for crack growth without remeshing. Int J Numer Method Eng 46(1):131-150

Mollenhauer D, Iarve EV, Kim R, Langley B (2006) Examination of ply cracking in composite laminates with open holes: A moiré interferometric and numerical study. Compos Part A 37(2):282-294

Moonen P, Sluys LJ, Carmeliet J (2008) A continuousdiscontinuous approach to simulate fracture processes in quasi-brittle materials. Philos Mag 88(28):3281-3298

Oliver J (2000) On the discrete constitutive models induced by strong discontinuity kinematics and continuum constitutive equations. Int J Solids Struct 37(48-50):7207-7229

Pinho ST, Robinson P, Iannucci L (2006) Physically based failure models and criteria for laminated fibre-reinforced composites with emphasis on fibre kinking. Part I: Development. Compos Part A 37(1):63-73

Puck A, Schürmann H (1998) Failure analysis of FRP laminates by means of physically based phenomenological models. Compos Sci Technol 58:1045-1067

Puck A, Schürmann H (2002) Failure analysis of FRP laminates by means of physically based phenomenological models. Compos Sci Technol 62:1633-1662

Rabczuk T, Zi G, Gerstenberger A, Wall WA (2008) A new crack tip element for the pantom node method with arbitrary cohesive cracks. Int J Numer Method Eng 75(5):577-599

Reeder JR, Crews JR Jr (1990) Mixed-mode bending method for delamination testing. AIAA J 28(7):1270-1276 
Song JH, Areias PMA, Belytschko T (2006) A method for dynamic crack and shear band propagation with phantom nodes. Int J Numer Method Eng 67(6):868-893

Tsai SW, Wu EM (1971) A general theory of strength for anisotropic materials. J Compos Mater 5:58-80

Turon A, Camanho PP, Costa J, Dávila CG (2006) A damage model for the simulation of delamination in advanced composites under variable-mode loading. Mech Mater 38(11):1072-1089

van der Meer FP, Sluys LJ (2009) Continuum models for the analysis of progressive failure in composite laminates. J Compos Mater (in print)

Van Paepegem W, De Baere I, Degrieck J (2006) Modelling the nonlinear shear stress-strain response of glass fibre-reinforced composites. Part I: Experimental results. Compos Sci Technol 66(10):1455-1464

Verhoosel CV, Remmers JJC, Gutiérrez MA (2008) A dissipation-based arc-length method for robust simulation of brittle and ductile failure. Int J Numer Method Eng 77:1290-1321
Wells GN, Sluys LJ (2001) A new method for modelling cohesive cracks using finite elements. Int J Numer Method Eng 50(12):2667-2682

Whitney JM, Browning CE, Hoogsteden W (1982) A double cantilever beam test for characterizing mode I delamination of composite materials. J Reinf Plast Compos 1(4):297-313

Wisnom MR, Chang FK (2000) Modelling of splitting and delamination in notched cross-ply laminates. Compos Sci Technol 60(15):2849-2856

Wisnom MR, Khan B, Hallett SR (2008) Size effects in unnotched tensile strength of unidirectional and quasi-isotropic carbon/epoxy composites. Compos Struct 84(1):21-28

Yang QD, Cox BN (2005) Cohesive models for damage evolution in laminated composites. Int J Fract 133(2):107-137 